Rafal Harat ${ }^{1}$, Ronika Alexander ${ }^{2}$, Sharon Gray ${ }^{2}$, Elane M. Gutterman ${ }^{3}$, Justyna Pluta ${ }^{4}$, Michael Pride ${ }^{5}$, Sebastian Shite ${ }^{5}$, Joanna Fijolek ${ }^{1}$, Jolanta Kozub ${ }^{1}$

'Chrzanów County Hospital, Chrzanów, Poland

${ }^{2}$ Pfizer Inc, Collegeville, PA, USA

${ }^{3}$ Via Research, LLC, Princeton Junction, NJ, USA

${ }^{4}$ Pfizer Inc, Warsaw, Poland (during the time of manuscript development)

${ }^{5}$ Pfizer Inc, Pearl River, NY, USA

\title{
Prospective, population-based surveillance of the burden of Streptococcus pneumoniae in community-acquired pneumonia in older adults, Chrzanów County, Poland, 2010 to 2012
}

This study and medical writing were supported by Pfizer Inc.

\section{Abstract}

Introduction: Community-acquired pneumonia (CAP) caused by Streptococcus pneumoniae is a substantial cause of morbidity and mortality among older adults. This study estimated incidences of CAP, chest X-ray-confirmed CAP (CXR+CAP), $S$ pneumoniae-positive CAP, $S$ pneumoniae-positive CXR +CAP, and $S$. pneumoniae serotype distribution among 46,000 at-risk adults aged $\geq 50$ years residing in Chrzanów County, Poland.

Material and methods: From January 2010 to January 2012, all facilities providing ambulatory and inpatient care enrolled all consenting resident patients with suspicion of CAP. Chest x-rays, urine, blood, and sputum samples were analyzed. Annualized incidence rates were determined. Presence of $S$ pneumoniae-positive CAP and/or $S$. pneumoniae serotype distribution was determined using the urine antigen detection assay (capable of detecting the serotypes in the 13-valent pneumococcal conjugate vaccine [PCV13]), BinaxNOW ${ }^{2}$, and/or microbiology cultures.

Results: Among 5055 enrolled patients, 1195 (23.7\%) were diagnosed with CAP and 1166 (23.4\%) had CXR+CAP. S. pneumoniae was detected in 144 (12.1\%) and 131 (11.2\%) patients from the CAP and CXR+CAP cohorts, respectively. Annualized incidence rates of CAP, CXR+CAP, S pneumoniae-positive CAP, and S. pneumoniae-positive CXR+CAP were 12.8, 12.5, 1.6, and 1.4 per 1000 residents, respectively. Among CXR+CAP patients, $39.7 \%$ were aged 50 to 64 years and $60.3 \%$ were aged $\geq 65$ years. Incidence rates generally increased with age. The most common serotypes in S. pneumoniae-positive CXR+CAP patients were $3(n=15), 23 F(n=10), 18 C(n=9)$, and $9 V(n=6)$. Conclusions: CAP due to PCV13 serotypes is a source of morbidity among adults $>50$ years and may be reduced by greater access to pneumococcal vaccines.

Key words: community-acquired pneumonia, Streptococcus pneumoniae, adults, Poland, CAP incidence

Pneumonol Alergol Pol 2016; 84: 95-103

\section{Introduction}

Pneumonia is a leading cause of death in adults worldwide[1, 2]; the incidence of pneumonia and hospitalization for pneumonia increases with age $[3,4]$. Similarly, the burden of community-acquired pneumonia (CAP), often caused by
Streptococcus pneumoniae $[1,4-7]$ is greater in older adult populations [8]. A review of primary articles reporting studies conducted in Europe, published from 1990 to 2007, assessing the clinical and economic burden of CAP found that incidence and associated mortality were highest in those $\geq 65$ years of age [9]. In Poland from 2008

Address for correspondence: Ronika Alexander, Pfizer Inc 500 Arcola Rd Collegeville, PA, USA, tel. (484) 865-6097, fax: 484-865-6418, e-mail: Ronika.Alexander@pfizer.com 10.5603/PiAP.2016.0007

Received: 11.11.2015

Copyright (C) 2016 PTChP

ISSN 0867-7077 
to 2009, all-cause, inpatient CAP increased with age, with rates of $1.5,4.0,8.8$, and 16.8 per 1000 adults aged 50 to 64,65 to 74,75 to 84 , and 85 to 99 years, respectively [10]; the case fatality rate for patients $\geq 50$ years with CAP was $18.6 \%$ [10]. In Polish adults from 2006 to 2009, incidence rates of invasive pneumococcal disease (IPD), which includes bacteremic CAP, were generally low in those $\geq 50$ years (from $0.07-1.93$ per 1000 inhabitants). The most common S. pneumoniae serotypes were $1,3,4,9 \mathrm{~V}, 9 \mathrm{~N}, 12 \mathrm{~F}, 14,19 \mathrm{~F}$, and $23 \mathrm{~F}$ in those $\geq 55$ years of age [11].

Although $S$. pneumoniae is a common cause of CAP, there are a dearth of epidemiologic data for the pneumococcal CAP burden in adults, and epidemiologic studies are often hampered by inadequate assessment methods. In Poland, disease incidence rates for IPD are based on cases identified via laboratory-confirmed surveillance systems (eg, cultures of blood and other sterile fluids). However, factors such as prior treatment with antibiotics may limit isolation of viable organisms, causing underestimation of incidence rates [12]. Furthermore, CAP associated with nonbacteremic or noninvasive pneumococcal disease is not fully captured by these methods, and no universal standard exists for the measure of noninvasive disease [13]. The commercially available BinaxNOW ${ }^{\circledast}$ S. pneumoniae Antigen Card (Alere Inc.,Waltham, MA) and the proprietary 13-valent multiplex urine antigen detection (UAD; Pfizer Inc, New York, NY) assay provide an opportunity to specifically assess the prevalence of noninvasive pneumococcal disease by allowing for the identification of nonbacteremic pneumonia cases through urine testing [14, 15]. Although BinaxNOW does not distinguish between $S$. pneumoniae serotypes, the UAD assay is capable of identifying serotypes specific to 13-valent pneumococcal conjugate vaccine (PCV13).

Thirteen-valent pneumococcal conjugate vaccine was approved in Europe for prevention of invasive disease and pneumonia caused by the corresponding $S$. pneumoniae serotypes in adults aged $\geq 18$ years and in the elderly [16]. PCV13 replaced 7-valent PCV (PCV7) due to the emergence of nonvaccine serotypes [17]. Recent data from approximately 85,000 patients from the Community-Acquired Pneumonia Immunization Trial in Adults (CAPiTA) supported the efficacy of PCV13 in adults aged $\geq 65$ years, demonstrating efficacy compared with placebo against vaccine-type CAP (including nonbacteremic CAP) and vaccine-type IPD [18].

Despite reported benefits of PCV13 in adults, vaccination is not widespread in Polish adults, in whom vaccine-preventable pneumococcal disease remains a factor. In adults aged $\geq 55$ years in Poland, $36 \%$ to $89 \%$ of IPD cases from 2006 to 2009 were caused by serotypes covered by 1 of the currently approved pneumococcal vaccines [11]. Despite the availability of PCVs in Poland since 2005, no national pneumococcal immunization efforts have been undertaken. It is now recommended that adults with chronic diseases and those $>50$ years receive PCV13 or 23-valent pneumococcal polysaccharide vaccine (PPSV23) [19]. However, PCV reimbursement is limited to children aged 2 months to 5 years who are at high risk for IPD or have chronic diseases; this factor likely contributes to PCV vaccination being largely confined to this subgroup [20]. Moreover, uptake of PPSV23 is historically low, with only $0.1 \%$ of Polish adults aged $>65$ years receiving $\geq 1$ PPSV23 dose from 2004 to 2006 [21]. Improved understanding of serotypes causing bacteremic and nonbacteremic CAP in Polish adults is necessary to guide vaccination policies. Thus, the primary objective of this study was to estimate prospective incidence of CAP, chest x-ray-confirmed CAP (CXR+CAP), $S$ pneumoniae-positive CAP, and $S$ pneumoniae-positive CXR+CAP among adults aged $\geq 50$ years in Chrzanów County, Poland, from 2010 to 2012. Secondary objectives were to describe $S$. pneumoniae serotype distribution and CAP fatality rates in this population.

\section{Material and methods}

\section{Study design and enrollment}

This active, prospective, population-based surveillance study of CAP in adults aged $\geq 50$ years (conducted from January 14, 2010, to January 13, 2012) included all healthcare facilities in Chrzanów County, Małpolskie, Poland, that provided ambulatory and inpatient care (1 hospital and 30 freestanding public and private clinics). Eligible patients included resident adults aged $\geq 50$ years with suspicion of CAP who were seeking healthcare.

The criteria used to select the surveillance area included a well-defined population of eligible patients; definable and limited number of study hospitals and outpatient clinics; high-quality in-hospital clinical microbiology testing; low rates ( $<5 \%$ per year) of migration; ability to diagnose and treat adults with severe disease associated with CAP; feasibility of obtaining information about healthcare use and healthcare system description (structure, referral, coordination/management); 
and ability of the investigator to implement and sustain CAP population-based surveillance during the course of a 2-year study period.

Community-acquired pneumonia was defined as infection of the alveoli, distal airways, and lung interstitium occurring outside the hospital. Patients were suspected of having CAP if they had $\geq 1$ of the following clinical signs or symptoms of respiratory illness: fever (temperature $\geq 38.0^{\circ} \mathrm{C}$ ) or hypothermia (temperature $\leq 35.5^{\circ} \mathrm{C}$ ) within 24 hours of enrollment, chills or rigors, pleuritic chest pain, cough, sputum production, dyspnea, tachypnea, malaise, or abnormal auscultatory findings suggestive of pneumonia. Patients were excluded if they were transferred to a participating healthcare facility after already being hospitalized for $\geq 48$ hours or had developed signs and symptoms of pneumonia after being hospitalized for $\geq 48$ hours.

Patients participated directly in the study for 1 day. Following enrollment, chest x-ray was performed; clinical specimens were collected; and patients were interviewed to obtain demographic and family information, medical history, and previous antibiotic use. A final diagnosis and vital status were confirmed $\leq 10$ days postenrollment; for patients hospitalized for $\geq 10$ days, vital status was assessed $\leq 120$ days postenrollment. The study was undertaken in accordance with the Declaration of Helsinki and International Conference on Harmonisation Guidelines for Good Clinical Practice and Good Epidemiological Practice. All enrolled patients underwent a standardized informed consent process.

\section{Study populations}

The overall CAP cohort included all patients with a final diagnosis of pneumonia. The CXR+CAP cohort included all patients with clinical pneumonia whose chest radiograph result was consistent with pneumonia as read by a radiologist. The S. pneumoniae-positive CAP cohort included all patients with a positive result for S. pneumoniae detected from culture (blood or acceptable-quality respiratory specimen [eg, sputum, bronchial wash]) or urine test (BinaxNOW $S$. pneumoniae Antigen Card [14] or a 13-valent multiplex UAD assay [15]). The $S$. pneumoniae-positive CXR + CAP cohort included all patients with clinical pneumonia who were $S$. pneumoniae-positive and whose chest radiograph result was consistent with pneumonia.

\section{Procedures}

Digitalized chest x-rays were performed and read by the principal investigator and 2 central readers at the inpatient location. Nasopharyngeal swabs and blood for bacteriologic culture were collected upon enrollment. Sputum was collected if clinically indicated. Other respiratory specimens (eg, tracheal aspirates, bronchial washing, pleural fluid) were collected for culture and Gram stain according to local standard of care. All respiratory and blood samples underwent bacterial culture at the local laboratory according to standard methodology for identification of $S$. pneumoniae and other pathogens. All S. pneumoniae isolates identified by the local laboratory were sent on transport media to a central laboratory for confirmation of identification and serotyping by the Quellung reaction.

Urine samples were tested using Pfizer's proprietary serotype-specific UAD assay, which detects $S$ pneumonia serotypes 1, 3, 4, 5, 6A/C, 6B, 7F/A, 9V/A, 14, 18C/A/B/F, 19A, 19F, and 23F [15]. Urine samples were also tested using the commercially available $S$. pneumoniae BinaxNOW assay.

\section{Statistical analysis}

Statistical analyses were descriptive. Incidence rate was defined as the number of new cases of CAP in the population aged $\geq 50$ years in the surveillance area divided by the total at-risk population aged $\geq 50$ years in the surveillance area. For the 2-year study period, aggregated annualized incidence rates were reported per 1000 residents per year with 95\% CI based on the Poisson distribution. Case fatality rate for the 2 years was expressed as the percentage of cases resulting in death during postenrollment follow-up among all persons diagnosed with CAP.

\section{Results}

\section{Study populations}

In 2010 and 2011, the estimated population of Chrzanów County, Poland, was 127,645 and 127,540 residents, respectively, and the at-risk population comprised 46,134 and 46,962 people, respectively, aged $\geq 50$ years. In total, 5055 patients were enrolled in the present study; however, 6 subjects did not have respiratory illness which resulted in a total of 5049 patients, of whom 1195 (23.7\%) were diagnosed with CAP (Table 1) and 1166 (23.4\%) had CXR+CAP. S. pneumoniae was detected in 144 (12.1\%) and 131 (11.2\%) patients from the CAP and CXR+CAP cohorts, respectively. The remaining 3854 (76.3\%) patients were found to have other non-CAP respiratory illnesses, the majority of which were lower respiratory tract infections (62.3\%; 3146/5049 patients). The majority of CAP 
Table 1. Patient demographics and clinical characteristics

\begin{tabular}{|c|c|c|c|}
\hline Variable & $\begin{array}{l}\text { Overall cohort* } \\
n=5055\end{array}$ & $\begin{array}{c}\text { CAP } \\
n=1195\end{array}$ & $\begin{array}{c}\text { S pneumoniae-positive CAP } \\
n=144\end{array}$ \\
\hline \multicolumn{4}{|l|}{ Age } \\
\hline Mean \pm SD, y & $65.2 \pm 10$ & $68.1 \pm 10.5$ & $68.3 \pm 11.4$ \\
\hline \multicolumn{4}{|l|}{ Years, $n(\%)$} \\
\hline $50-64$ & $2653(52.5)$ & $481(40.3)$ & $58(40.3)$ \\
\hline $65-69$ & $645(12.8)$ & $161(13.5)$ & $21(14.6)$ \\
\hline $70-79$ & $1263(25.0)$ & $355(29.7)$ & $36(25.0)$ \\
\hline$\geq 80$ & $494(9.8)$ & $198(16.6)$ & $29(20.1)$ \\
\hline \multicolumn{4}{|l|}{ Sex } \\
\hline Female & $2911(57.6)$ & $619(51.8)$ & $69(47.9)$ \\
\hline Male & $2144(42.4)$ & $576(48.2)$ & $75(52.1)$ \\
\hline \multicolumn{4}{|c|}{ Previous enrollment in study } \\
\hline Yes & $334(6.6)$ & $120(10.0)$ & $18(12.5)$ \\
\hline \multicolumn{4}{|c|}{ Hospitalized for pneumonia in the past 5 years } \\
\hline No & $4680(92.6)$ & $1058(88.5)$ & $116(80.6)$ \\
\hline Yes & $364(7.2)$ & $137(11.5)$ & $28(19.4)$ \\
\hline Unknown & $11(0.2)$ & 0 & 0 \\
\hline \multicolumn{4}{|l|}{ Previous $\mathrm{PCV}^{\dagger}$} \\
\hline No & NA & $1191(99.7)$ & $143(99.3)$ \\
\hline Yes & NA & 0 & 0 \\
\hline Unknown & NA & $4(0.3)$ & $1(0.7)$ \\
\hline \multicolumn{4}{|l|}{ Previous $\mathrm{PPV}^{\dagger}$} \\
\hline No & NA & $1195(100)$ & $144(100)$ \\
\hline Yes & NA & 0 & 0 \\
\hline Unknown & NA & 0 & 0 \\
\hline \multicolumn{4}{|c|}{ Previous influenza vaccine } \\
\hline No & NA & $1080(90.4)$ & $131(91.0)$ \\
\hline Yes & NA & $112(9.4)$ & $12(8.3)$ \\
\hline Unknown & NA & $3(0.3)$ & $1(0.7)$ \\
\hline \multicolumn{4}{|l|}{ Smoking history } \\
\hline Current smoker & NA & $235(19.7)$ & $44(30.6)$ \\
\hline Former smoker & NA & $513(42.9)$ & $59(41.0)$ \\
\hline Never smoked & NA & $447(37.4)$ & $41(28.5)$ \\
\hline
\end{tabular}

patients were current (19.7\%) or former (42.9\%) smokers, only $9.4 \%$ had received an influenza vaccine within the past year, and none had previously received a pneumococcal vaccine.

Of patients diagnosed with CAP, $40.3 \%$ were aged 50 to 64 years and 59.8\% were aged $\geq 65$ years (Table 1). Almost $40 \%$ of CAP patients were hospitalized, and the remaining patients required only an emergency department stay or outpatient care. For hospitalized patients, the mean \pm SD length of stay was $10.9 \pm 7.1$ days. Nearly all cases of CAP (97.6\%) were radiographically confirmed 


\section{Table 2. Incidence rates of pneumonia}

\begin{tabular}{|c|c|c|c|}
\hline Diagnosis & Number of cases* & $\begin{array}{c}\text { Number } \\
\text { in at-risk } \\
\text { population }\end{array}$ & $\begin{array}{c}\text { Incidence rate per } \\
1000 \text { population } \\
\text { (95\% Cl) }\end{array}$ \\
\hline CAP & 1195 & 93,096 & $12.8(12.1-13.6)$ \\
\hline \multicolumn{4}{|l|}{ Age groups, y } \\
\hline $50-64$ & 481 & 55,780 & $8.6(7.9-9.4)$ \\
\hline$\geq 65$ & 714 & 37,316 & $19.13(17.7-20.6)$ \\
\hline $65-69$ & 161 & 9907 & $16.3(13.8-19.0)$ \\
\hline $70-79$ & 355 & 19,082 & $18.6(16.7-20.6)$ \\
\hline$\geq 80$ & 198 & 8327 & $23.8(20.6-27.3)$ \\
\hline $\mathrm{CXR}+\mathrm{CAP}$ & 1166 & 93,096 & $12.5(11.8-13.3)$ \\
\hline \multicolumn{4}{|l|}{ Age groups, y } \\
\hline $50-64$ & 469 & 55,780 & $8.4(7.7-9.2)$ \\
\hline$\geq 65$ & 697 & 37,316 & $18.7(17.3-20.1)$ \\
\hline $65-69$ & 156 & 9907 & $15.8(13.4-18.4)$ \\
\hline $70-79$ & 346 & 19,082 & $18.1(16.3-20.2)$ \\
\hline$\geq 80$ & 195 & 8327 & $23.4(20.3-27.0)$ \\
\hline S. pneumoniae-positive CAP & 144 & 93,096 & $1.6(1.3-1.8)$ \\
\hline \multicolumn{4}{|l|}{ Age groups, y } \\
\hline $50-64$ & 58 & 55,780 & $1.0(0.8-1.3)$ \\
\hline$\geq 65$ & 86 & 37,316 & $2.3(1.8-2.9)$ \\
\hline $65-69$ & 21 & 9907 & $2.1(1.3-3.2)$ \\
\hline $70-79$ & 36 & 19,082 & $1.9(1.3-2.6)$ \\
\hline$\geq 80$ & 29 & 8327 & $3.5(2.3-5.0)$ \\
\hline S. pneumoniae-positive CXR +CAP & 131 & 93,096 & $1.4(1.2-1.7)$ \\
\hline \multicolumn{4}{|l|}{ Age groups, $y$} \\
\hline $50-64$ & 52 & 55,780 & $0.9(0.7-1.2)$ \\
\hline$\geq 65$ & 79 & 37,316 & $2.1(1.7-2.6)$ \\
\hline $65-69$ & 17 & 9907 & $1.7(1.0-2.8)$ \\
\hline $70-79$ & 33 & 19,082 & $1.7(1.2-2.4)$ \\
\hline$\geq 80$ & 29 & 8327 & $3.5(2.3-5.0)$ \\
\hline
\end{tabular}

CAP - community-acquired pneumonia; CXR + CAP — chest $x$-ray-confirmed CAP

${ }^{*}$ Annualized data from both study years

(CXR+CAP; Table 2); $40.3 \%$ and $59.7 \%$ of CAP patients and $40.2 \%$ and $59.8 \%$ of CXR +CAP patients were aged 50 to 64 and $\geq 65$ years, respectively. More than $50 \%$ of patients with $S$. pneumoniae -positive CAP were hospitalized, and the length of stay was $10.6 \pm 5.6$ days. More than $90 \%$ of S. pneumoniae-positive CAP patients (131/144 patients) were also CXR positive (Table 2). A total of $11.2 \%(131 / 1166)$ patients with CXR+CAP were $S$. pneumoniae positive, $62.6 \%$ of whom were former or current smokers. More than half of patients with $S$. pneumoniae-positive CXR+CAP were hospitalized and the length of stay was $10.4 \pm 5.4$ days.

\section{Incidence rates}

The annualized incidence rate of CAP and CXR+CAP in at-risk adults was 12.8 and 12.5 per 1000 residents, respectively, and rose to 19.1 and 18.7 per 1000 residents in adults aged $\geq 65$ years (Table 2). The incidence of $S$. pneumoniae-positive $\mathrm{CAP}$ and $S$. pneumoniae-positive CXR +CAP in 
adults aged $<65$ years was 1.6 and 1.4 per 1,000 residents, respectively, and incidence increased to 2.3 and 2.1 per 1000 residents in adults aged $\geq 65$ years (Table 2). Overall, the incidence of CAP, CXR + CAP, S. pneumoniae-positive CAP, and S. pneumoniae-positive CXR + CAP increased with age. Results across all cohorts were similar in both study years.

\section{Case fatality}

Nineteen $(1.6 \%)$ patients with CAP died in the follow-up period. Deaths were distributed across age groups (5 were aged 50-64 years; 14 were aged $\geq 65$ years). Of these 19 patients, 18 (1.5\%) had CXR+CAP; S. pneumoniae was identified in 1 patient.

\section{Streptococcus pneumoniae culture from blood and sputum samples}

All 1195 patients with CAP had blood cultures; none grew $S$. pneumoniae. Among CAP patients, $21.4 \%$ reported antibiotic use in the week before enrollment; 7 had $S$. pneumoniae confirmed by the central laboratory from sputum samples and 8 from bronchial wash/bronchoalveolar lavage samples. All but 1 patient with CAP had a nasopharyngeal swab, and 7 patients (2.4\%) had swabs that grew $S$. pneumoniae.

\section{Urine analyses for Streptococcus pneumoniae}

A UAD and/or BinaxNOW assay was performed on urine samples obtained from $98.3 \%$ of enrolled patients (4971 and 4970, respectively). A total of 107 patients had an S. pneumoniae-positive UAD result, and 69 of these patients had CAP. Of the 242 patients with an S. pneumoniae-positive BinaxNOW result, 97 had CAP. Few patients (32 of 1195 with CAP [2.7\%]) had both a positive UAD and BinaxNOW assay result. Three patients were positive by sputum and BinaxNOW and/or UAD, and 3 were positive by lavage and BinaxNOW and/or UAD.

\section{Streptococcus pneumoniae serotypes}

Of the 144 patients with $S$. pneumoniae-positive CAP, 76 had PCV13 serotypes, 1 had a non-PCV13 serotype (serotype 9N), 1 was not serotyped, and 66 were positive by BinaxNOW only. All but 1 of the 68 serotypes identified in patients with $S$. pneumoniae-positive CXR +CAP were PCV13 serotypes; the only non-PCV13 serotype was $9 \mathrm{~N}$ (Table 3 ). The most common serotypes identified were $3(n=15), 23 F(n=10)$,
18C $(\mathrm{n}=9)$, and 9V $(\mathrm{n}=6$; Table 3$)$. Serotypes identified included PCV7 serotypes 14 and 23F (1 patient each); additional PCV13 serotype 1 (2 patients); and non-PCV13 serotypes 9N, 11A, and 23B (1 patient each).

\section{Discussion}

A burden of CAP and S. pneumoniae-positive CAP existed in older adults in Chrzanów County, Poland, during study years 1 and 2, and for the annualized results. Typical for most of Poland, pneumococcal vaccination in Chrzanów is rare among adults [21] and limited to young children with high-risk conditions. CAP incidence in patients aged $\geq 65$ years (19.13 per 1000 residents) was similar to that found in a retrospective assessment of CAP burden in Kielce, Poland (19.4 per 1000 residents) before the introduction of a municipal PCV7 vaccination program in young children [3]. The annual incidence rate of CXR+CAP in rural Crete, Greece was 2.4 per 1000 residents, lower than that observed in the current study (12.5 per 1000), potentially due to differences in disease incidence or variations in study design/data collection [22].

Most serotypes in S. pneumoniae-positive CAP patients were identified using the UAD assay and, thus, included only PCV13 serotypes. The most frequently identified serotypes were $3,23 \mathrm{~F}$, $18 \mathrm{C}$, and 9V. Only 1 non-PCV13 serotype (9N) was identified. The lack of non-PCV13 serotypes is a result of an unexpectedly low culture yield as opposed to any change in the prevalence of specific serotypes. In a previous Polish study conducted from 2006 to 2009 among adults aged $\geq 55$ years with IPD, serotypes 3 and $23 \mathrm{~F}$ were among the most common, whereas serotypes 18C and $6 \mathrm{~A}$ were less common [11]. These differences may reflect differing etiologies of noninvasive versus invasive disease or the relative sensitivities of the different bacterial identification methods. For example, validation studies for the UAD assay demonstrated $97 \%$ sensitivity for the 13 PCV13 serotypes compared with traditional blood culture [15].

Earlier studies demonstrated the utility of an assay similar to the UAD assay in the identification of serotypes associated with noninvasive CAP [23, 24]. Differences in the prevalence of serotypes identified in urine samples compared with those identified in cases of bacteremic disease in 2 hospitals in the United Kingdom have been reported [23]. The most prevalent serotype found in urine 
Table 3. S pneumoniae serotypes among subjects with CXR+CAP, and $S$. pneumonia-positive non-CXR+CAP and other respiratory infection*

\begin{tabular}{|c|c|c|c|c|}
\hline \multirow[b]{2}{*}{ Serotype $^{\dagger}$} & \multicolumn{4}{|c|}{$\begin{array}{c}\text { S. pneumonia-positive } \\
\text { Cases (\%) }\end{array}$} \\
\hline & $\begin{array}{c}\text { CXR+CAP } \\
(n=131) \\
\text { serotyped } n=67\end{array}$ & $\begin{array}{c}\text { non-CXR + CAP } \\
(n=13) \\
\text { serotyped } n=10\end{array}$ & $\begin{array}{c}\text { Other respiratory } \\
\text { infection }^{\ddagger}(n=177) \\
\text { serotyped } n=38\end{array}$ & $\begin{array}{c}\text { Total } \\
(n=321) \\
\text { serotyped } n=115\end{array}$ \\
\hline \multicolumn{5}{|c|}{ PCV7 serotypes } \\
\hline 4 & $5(7.5)$ & 0 & $1(2.6)$ & $6(5.2)$ \\
\hline $6 \mathrm{~B}$ & $5(7.5)$ & $3(30)$ & $4(10.5)$ & $12(10.4)$ \\
\hline $9 \mathrm{~V}$ & $6(9.0)$ & 0 & $2(5.3)$ & $8(7.0)$ \\
\hline 14 & $5(7.5)$ & 0 & $12(31.6)$ & $17(14.8)$ \\
\hline $18 \mathrm{C}$ & $9(13.4)$ & $1(10)$ & $7(18.4)$ & $17(14.8)$ \\
\hline $19 \mathrm{~F}$ & $3(4.5)$ & $1(10)$ & $1(2.6)$ & $5(4.3)$ \\
\hline $23 \mathrm{~F}$ & $10(14.9)$ & $1(10)$ & $5(13.2)$ & $16(13.9)$ \\
\hline $\begin{array}{l}\text { All PCV7 serotypes } \\
\text { combined }\end{array}$ & $43(64.2)$ & $6(60)$ & $32(84.2)$ & $81(70.4)$ \\
\hline \multicolumn{5}{|c|}{ PCV13 serotypes } \\
\hline \multicolumn{5}{|c|}{6 additional serotypes in PCV13 } \\
\hline 1 & $1(1.5)$ & $3(30)$ & $1(2.6)$ & $5(4.3)$ \\
\hline 3 & $15(22.4)$ & 0 & 0 & $15(13.0)$ \\
\hline 5 & $1(1.5)$ & $1(10)$ & $4(10.5)$ & $6(5.2)$ \\
\hline $6 \mathrm{~A}$ & $4(6.0)$ & 0 & 0 & $4(3.5)$ \\
\hline $7 F$ & $1(1.5)$ & 0 & $1(2.6)$ & $2(1.7)$ \\
\hline $19 A$ & $2(3.0)$ & 0 & $1(2.6)$ & $3(2.6)$ \\
\hline $\begin{array}{l}6 \text { additional PCV13 se- } \\
\text { rotypes combined }\end{array}$ & $23(34.3)^{5}$ & $4(40)$ & $7(18.4)$ & $34(29.6)$ \\
\hline $\begin{array}{l}\text { All PCV13 serotypes } \\
\text { combined }\end{array}$ & $66(98.5)^{5}$ & $10(100)$ & $38(100)$ & $114(99.1)^{\S}$ \\
\hline \multicolumn{5}{|c|}{ Non-PCV13 serotypes } \\
\hline $9 \mathrm{~N}$ & $1(1.5)$ & 0 & 0 & $1(.9)$ \\
\hline $\begin{array}{l}\text { BinaxNOW-only } \\
\text { positive }\end{array}$ & 64 (NA) & 2 (NA) & 139 (NA) & 205 (NA) \\
\hline \multicolumn{5}{|c|}{$\begin{array}{l}\text { CAP — community-acquired pneumonia; CXR — chest } x \text {-ray; NA — not applicable; PCV7 - 7-valent pneumococcal conjugate vaccine; PCV13 - 13-valent pneumo } \\
\text { coccal conjugate vaccine; UAD - urine antigen detection } \\
{ }^{*} \text { Annualized data from both study years } \\
{ }^{+} \text {Results based on blood, respiratory, and urine samples. Isolates from respiratory specimens were serotyped using the Quellung reaction. Urine samples were serotypec } \\
\text { using the UAD assay } \\
{ }^{\ddagger} \text { Includes those with other nonpneumonia respiratory diseases } \\
{ }^{\S} \text { One subject had more than } 1 \text { unique serotype result: } 6 \mathrm{~A} \text { and } 19 \mathrm{~A}\end{array}$} \\
\hline
\end{tabular}

samples collected in support of that study was serotype 14. This serotype was not represented within the bacteremic group, suggesting that its prevalence may have been underestimated [23]. Similar results using the same UAD as the current study have been reported [24]. These data suggest that serotype distribution measured in bacteremic pneumonia may not represent the serotypes causing noninvasive pneumonia in those same groups.
The current analysis has inherent strengths and limitations. Clear strengths lie within its design as a prospective, population-based study the gold standard in burden of disease analysis. Moreover, the surveillance method provided a comprehensive assessment of disease among older adults in Chrzanów County, Poland, by systematically enrolling all patients with suspected CAP from all primary care clinics and the 1 hospital in the county. Based on the surveil- 
lance estimate, the overall proportion of eligible subjects enrolled was 92\% (95\% CI, 91.3-92.7); the number of patients aged $>50$ years registered for primary care in the county ( $\mathrm{n}=45,356$ as of January 1, 2010) was $98.3 \%$ of the estimated county population within these age groups $(\mathrm{n}=$ 46,134; population as of December 31, 2009). This congruence suggests that almost all adult inhabitants of Chrzanów County received their medical care within the county, although this was not confirmed with a survey. Finally, S. pneumoniae infection and associated serotypes were identified using the UAD assay, allowing for the detection of nonbacteremic pneumonia cases using urine testing and providing more specific information on $S$. pneumoniae serotypes associated with noninvasive disease.

Study limitations include the fact that it was conducted in only 1 county in Poland; consequently, results may not be widely generalizable. However, a prior hospital-based study using electronic administrative data comparing Chrzanów and Inowrocław County hospitals from 2006 to 2008 found a higher incidence of hospitalization for pneumonia in Inowrocław County in most age groups, including older adults [25]. Among the 1077 eligible hospitalized patients, $26.5 \%$ were not enrolled, primarily due to mental incapacity to provide informed consent. Therefore, CAP incidence may be underestimated. Further complicating estimates of $S$. pneumoniae incidence, almost $25 \%$ of patients with clinical pneumonia self-reported antibiotic treatment in the week before enrollment. This may have reduced viable bacteria yield in blood and respiratory specimens. Although lack of positive blood cultures was unexpected, assessment with UAD and BinaxNOW assays permitted identification of $S$. pneumoniae infections not identified by blood culture.

\section{Conclusions}

Despite limited epidemiologic data on pneumococcal CAP in Poland, trends of population aging and increased morbidity in the elderly [3,4] in association with the cost of treatment, support the need to prevent CAP in Polish adults with an effective vaccine. Results of this prospective, population-based study suggest that pneumonia burden in Poland is substantial and is consistent with previous analyses [8]. Continued surveillance of noninvasive CAP is required to better understand which serotypes are responsible for causing disease in this setting.
Additionally, greater rigor in culture methods might affect yield in future studies. Despite these challenges, CAP due to PCV13 serotypes clearly remains a source of morbidity among adults in Chrzanów County. PCV13 vaccination of older adults, along with broader vaccination of infants, has the potential to reduce pneumococcal disease incidence in Poland.

\section{Acknowledgments}

We acknowledge the MediScience team and Marcin Ossowski for their operational and administrative support, assistance in study implementation and data acquisition, and site communication (MediScience Network, Warsaw, Poland). Editorial/medical writing support was provided by Nicole Gudleski O'Regan, PhD, of Complete Healthcare Communications, LLC (Chadds Ford, PA).

\section{Conflict of interest}

EMG, President of Via Research, LLC, was hired by the sponsor as a consultant to provide epidemiologic support and conduct quality assurance assessments during the study. RH, JF, and JK have nothing to disclose. MP, JP, RA, SG, and SS are employees of Pfizer.

\section{References:}

1. World Health Organization. 23-valent pneumococcal polysaccharide vaccine. WHO position paper. Wkly Epidemiol Rec 2008; 83: 373-384.

2. World Health Organization. Global Burden of Disease 2004: Part 2, Causes of Death. Available at: http://www.who.int/healthinfo/global_burden_disease/GBD_report_2004update_part2. pdf; 12.02 .2015 .

3. Patrzalek M, Gorynski P, Albrecht P. Indirect population impact of universal PCV7 vaccination of children in a $2+1$ schedule on the incidence of pneumonia morbidity in Kielce, Poland. Eur J Clin Microbiol Infect Dis 2012; 31: 3023-3028. doi: 10.1007/s10096-012-1656-0.

4. Gil Prieto R, Garcia-Garcia L, Alvaro-Meca A, Mendez C, Garcia A, de Miguel AG. The burden of hospitalisations for community-acquired pneumonia (CAP) and pneumococcal pneumonia in adults in Spain (2003-2007). Vaccine 2011; 29: 412-416. doi: 10.1016/j.vaccine.2010.11.025.

5. Sanz Herrero F, Blanquer Olivas J. Microbiology and risk factors for community-acquired pneumonia. Semin Respir Crit Care Med 2012; 33: 220-231. doi: 10.1055/s-0032-1315634.

6. World Health Organization. Initiative for Vaccine Research (IVR): Acute Respiratory Infections (Update September 2009) - Streptococcus pneumoniae. Available at: http://apps.who. int/vaccine_research/diseases/ari/en/index3.html; 12.02.2015.

7. van Mens SP, Meijvis SC, Endeman $\mathrm{H}$ et al. Longitudinal analysis of pneumococcal antibodies during community-acquired pneumonia reveals a much higher involvement of Streptococcus pneumoniae than estimated by conventional methods alone. Clin Vaccine Immunol 2011; 18: 796-801. doi: 10.1128/ CVI.00007-11.

8. Torres A, Peetermans WE, Viegi G, Blasi F. Risk factors for community-acquired pneumonia in adults in Europe: a li- 
terature review. Thorax 2013; 68: 1057-1065. doi: 10.1136/ thoraxjnl-2013-204282.

9. Welte T, Torres A, Nathwani D. Clinical and economic burden of community-acquired pneumonia among adults in Europe. Thorax 2012; 67: 71-79. doi: 10.1136/thx.2009.129502.

10. Tichopad A, Roberts C, Gembula I et al. Clinical and economic burden of community-acquired pneumonia among adults in the Czech Republic, Hungary, Poland and Slovakia. PLoS ONE 2013; 8: e71375. doi: 10.1371/journal.pone.0071375

11. Skoczynska A, Sadowy E, Bojarska K et al. The current status of invasive pneumococcal disease in Poland. Vaccine 2011; 29: 2199-2205. doi: 10.1016/j.vaccine.2010.09.100.

12. Werno AM, Murdoch DR. Medical microbiology: laboratory diagnosis of invasive pneumococcal disease. Clin Infect Dis 2008; 46: 926-932. doi: 10.1086/528798.

13. Said MA, Johnson HL, Nonyane BA et al. Estimating the burden of pneumococcal pneumonia among adults: a systematic review and meta-analysis of diagnostic techniques. PLoS ONE 2013; 8: e60273. doi: 10.1371/journal.pone.0060273.

14. Murdoch DR, Laing RT, Mills GD et al. Evaluation of a rapid immunochromatographic test for detection of Streptococcus pneumoniae antigen in urine samples from adults with community-acquired pneumonia. J Clin Microbiol 2001; 39: 3495-3498.

15. Pride MW, Huijts SM, Wu K et al. Validation of an immunodiagnostic assay for detection of 13 Streptococcus pneumoniae serotype-specific polysaccharides in human urine. Clin Vaccine Immunol 2012; 19: 1131-1141. doi: 10.1128/CVI.00064-12.

16. European Medicines Agency. Prevenar 13. Available at: http://www.emea.europa.eu/ema/index.jsp?curl = pages/medicines/human/medicines/001104/human_med_001220.jsp; 27.10.2013.

17. Weinberger DM, Malley R, Lipsitch M. Serotype replacement in disease after pneumococcal vaccination. Lancet 2011; 378: 1962-1973. doi: 10.1016/S0140-6736(10)62225-8.
18. Bonten MJ, Huijts SM, Bolkenbaas M et al. Polysaccharide conjugate vaccine against pneumococcal pneumonia in adults. N Engl J Med 2015; 372: 1114-1125. doi: 10.1056/NEJMoa1408544.

19. Albrecht P, Antczak A, Hryniewicz W et al. Recommendations for prevention of community-acquired pneumonia with bacteremia as the leading form of invasive pneumococcal infections in the population of people over 50 years of age and risk groups above 19 years of age. Pol Merkur Lekarski 2014; 36: 79-87.

20. Posobkiewicz M. Program Szczepień Ochronnych na rok 2015. Available at: http://dziennikmz.mz.gov.pl/DUM_MZ/2014/72/ akt.pdf; 12.02.2015.

21. Nitsch-Osuch A, Wardyn AK, Choroszy-Krol I. Pneumococcal and influenza vaccine coverage in persons aged $>65$ years in 2004-2006 in Poland. Fam Med Prim Care Rev 2008; 10: $578-580$.

22. Bertsias A, Tsiligianni IG, Duijker G, Siafakas N, Lionis C, Cretan CAPRG. Studying the burden of community -acquired pneumonia in adults aged $\geq 50$ years in primary health care: an observational study in rural Crete, Greece. NPJ Prim Care Respir Med 2014; 24: 14017. doi: 10.1038/ npjpcrm.2014.17.

23. Bewick T, Sheppard C, Greenwood S et al. Serotype prevalence in adults hospitalised with pneumococcal non-invasive community-acquired pneumonia. Thorax 2012; 67: 540-545. doi: 10.1136/thoraxjnl-2011-201092.

24. Sherwin RL, Gray S, Alexander R et al. Distribution of 13-valent pneumococcal conjugate vaccine Streptococcus pneumoniae serotypes in US adults aged $\geq 50$ years with community -acquired pneumonia. J Infect Dis 2013; 208: 1813-1820. doi: 10.1093/infdis/jit506.

25. Harat R, Gorny G, Jorgensen L et al. A retrospective study of hospitalized pneumonia in two Polish counties $(2006-2008)$. Pneumonol Alergol Pol 2013; 81: 429-438. 